\title{
Non-Emergent Early Surgical Management for Adhesive Small Bowel Obstruction
}

\author{
Sawsan J. Horani Labib S. Al-Ozaibi Hadiel A. Kaiyasah \\ General Surgery Department, Rashid Hospital, Dubai Health Authority, Dubai, UAE
}

\section{Keywords}

Adhesive small bowel obstruction - Early surgery ·

Emergency · Gastroenterology · General surgery · Surgery ·

Treatment

\begin{abstract}
Background: Adhesive small bowel obstruction (ASBO) is one of the leading reasons for emergency surgical admissions. Current guidelines recommend the conservative approach, unless operative intervention is indicated. Case: Here, we present a case of a young male who underwent early laparoscopic adhesiolysis for ASBO. Discussion: Nonoperative management resolves majority of ASBO cases but has high recurrence. Early surgical management (ESM) has been shown to reduce recurrence rate and long-term health consequences. Conclusion: ESM can be considered for recurrent ASBO in younger patients with fewer comorbidities.

$$
\begin{aligned}
& \text { (c) } 2021 \text { The Author(s) } \\
& \text { Published by S. Karger AG, Basel }
\end{aligned}
$$
\end{abstract}

requirement of emergent surgical exploration to rule out compromised bowel, permitting a trial of conservative management [1]. In view of high morbidity and mortality associated with emergency surgery in the setting of adhesive small bowel obstruction (ASBO), it should be only considered when signs of strangulation, peritonitis, or bowel ischemia are present [2]. Recently, many studies have advocated for early surgical management (ESM) under the tenet that it incurs more health benefits and lower cost expenditure for patients on the long run [2]. Surgical intervention is still believed to lead to further adhesions and increase the risk of bowel resection and incisional hernia [2-4]. Though evidence is still conflicting, the diminished recurrence rates and long-term health benefits ensuing ESM has earned this alternative approach momentum among many surgeons today $[2,4]$. For the purpose of this publication, we restrict our definition of recurrent ASBO to readmissions following complete resolution of the previous episode of ASBO. Our aim is to report a case of ASBO managed by early non-emergent laparoscopic intervention.

\section{Case Report}

A 30-year-old male presented to our Emergency Department following traumatic fall from the third floor. Patient had sustained multiple fractures involving the pelvis and extremities, as well as abdominal injuries for which he underwent pelvic fixation and 2 surgical training was to "never let the sun rise or set on small bowel obstruction." Since then, the increasing availability of computed tomography (CT) has supplanted the

karger@karger.com www.karger.com/dmj

Karger $\stackrel{\text { ' }}{5}$

GOPEN ACCESS
C 2021 The Author(s)

Published by S. Karger AG, Basel

This is an Open Access article licensed under the Creative Commons Attribution-NonCommercial-4.0 International License (CC BY-NC) (http://www.karger.com/Services/OpenAccessLicense), applicable to the online version of the article only. Usage and distribution for commercial purposes requires written permission.
Correspondence to:

Sawsan J. Horani, horanisawsan@gmail.com 


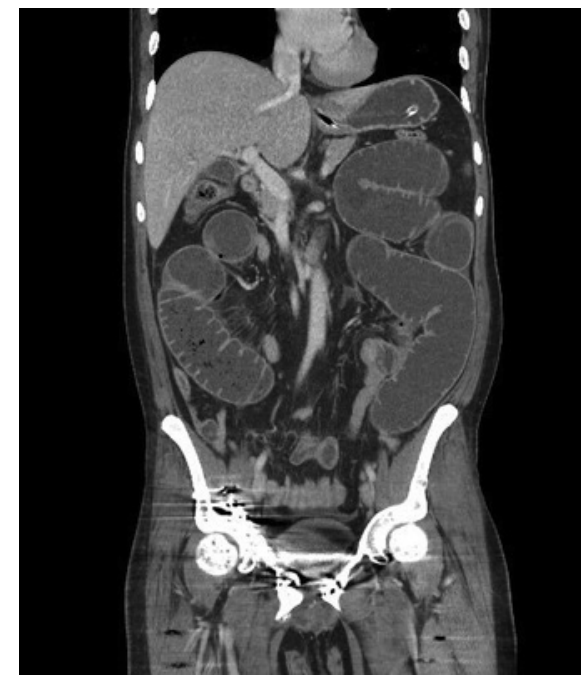

Fig. 1. Coronal computed tomography showing dilation of small bowel with transition zone in left lumbar region.

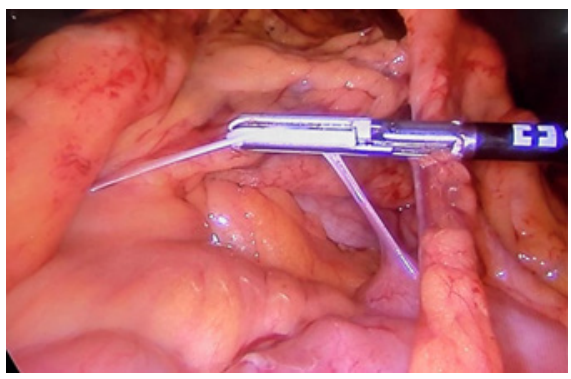

Fig. 3. Adhesive band identified in laparoscopic surgery.

laparotomies. First explorative laparotomy revealed minimal blood in abdominal cavity, pelvic and sigmoid mesenteric hematoma, as well as right liver lobe lacerations. Upon relook $48 \mathrm{~h}$ later, the second laparotomy revealed liver lacerations with resolving pelvic and sigmoid mesenteric hematomas and intact small and large bowel. Patient was discharged in stable condition.

Three weeks later, the patient returned to the Emergency Department with non-radiating abdominal pain of 3 days duration, associated with fever, vomiting, bloating, and obstipation. Physical examination revealed distended abdomen without peritoneal signs. Laboratory results including inflammatory marker and lactic acid levels were within normal limits. Chest X-ray was normal. Abdominal X-ray revealed dilated loops in the left upper quadrant. Abdominopelvic CT showed dilated jejunal and ileal loops with a transition zone in the left lumbar region (Fig. 1). Patient was admitted for ASBO. He improved on nonoperative management (NOM) and was tolerating gradual progression of diet with no complaints, so he was discharged with follow-up appointment in 3 weeks. Two weeks later, patient revisited ER for similar complaints of severe colicky abdominal pain of 1-day duration. Physical examination revealed distended abdomen without peritoneal signs. Laboratory results returned within normal limits. Abdominal X-ray revealed multiple air-fluid levels with dilated small bowel loops. Abdomino-

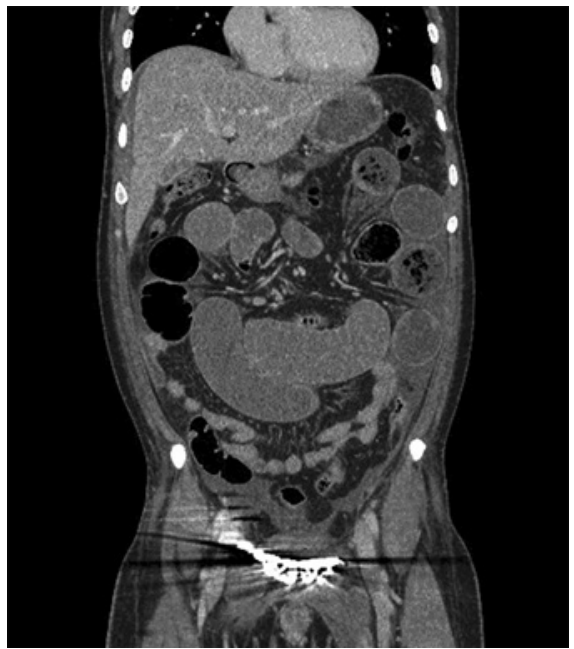

Fig. 2. Coronal cut showing collapsed loops, transition zone, and free fluid.

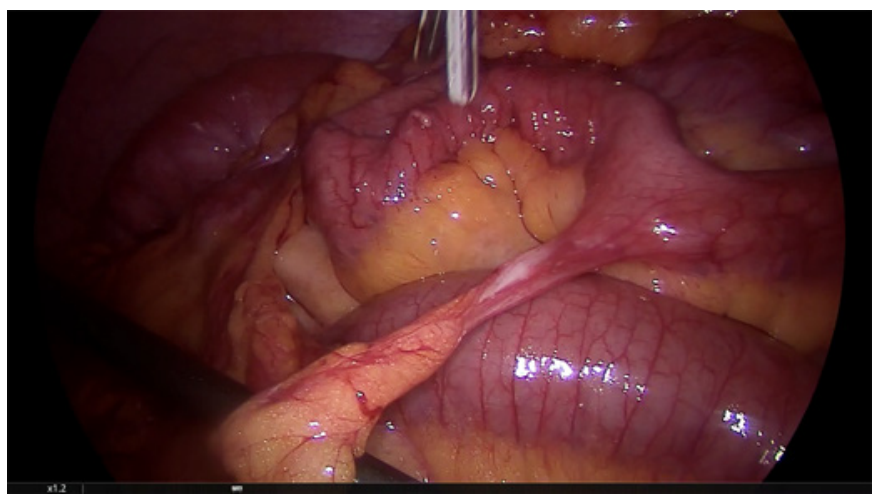

Fig. 4. Intraoperative image showing adhesive band.

pelvic CT confirmed mechanical obstruction with transition zone (Fig. 2). He improved on NOM, the obstruction was relieved, and diet was gradually advanced from oral clear fluid followed by liquid, soft, and then full diet as per patient's tolerance.

In view of repeated admissions of obstruction in a short period of time, the decision was made to operate to decrease the chance of recurrence. Pneumoperitoneum was created by open technique and intraoperative findings of diagnostic laparoscopy showed adhesive bands, which were released (Fig. 3, 4). Postoperative recovery was uneventful.

\section{Discussion}

Adhesiogenesis is a form of pathologic healing response of peritoneal injury that starts immediately during surgery and results in fibrinous attachments between surfaces, as opposed to the "ad integrum" normal repair [4]. 
If fibrinolysis does not take place within a week of peritoneal injury, the temporary fibrin matrix becomes a scaffold and peritoneal adhesions form $[5,6]$. Postoperative adhesions instigate $60-75 \%$ of small bowel obstructions $[2-4,7]$. This risk is highest following colorectal, oncologic, gynecologic, and pediatric operations $[2,7]$. Although NOM can ameliorate symptoms, relieve obstruction, and restore function, it does not eliminate the offending adhesions, which can provoke the recurrence of ASBO [5]. While NOM is effective in $70-90 \%$ of cases, it is associated with high readmission rates $(20-35 \%)[2,4$, 7]. A longitudinal population-based study comparing ESM and NOM for recurrence rates revealed a significant difference of 13 versus $21.3 \%$. In fact, the recurrence of ASBO increased with every episode until surgical management, which subsequently curtailed recurrence by $50 \%$. It is important to note that those treated operatively were younger ( $<80$ years) and had a lower comorbidity burden [4].

Therefore, ASBO might be better perceived as a chronic relapsing surgical disease. As studies show that recurrence is frequent and, with each episode, the risk of recurrence escalates while the time between attacks declines. Avoiding surgery may fail to account for the relapsing nature of the disease. Moreover, operative management of the first episode of ASBO has been shown to have a preventive effect for future recurrences compared to NOM [4].

Compared to laparotomy, the laparoscopic approach has proven to be less adhesiogenic and is associated with less postoperative pain, shorter hospital stays, minor complications, earlier refeeding, and lower reoperation rates (1.4 vs. 3.8\%) [5, 8]. When feasible, studies endorse ESM as it is associated with earlier regain of intestinal function and fewer radiological exams [5].

\section{Conclusion}

Current guidelines recommend a trial of NOM lasting at least $72 \mathrm{~h}$ before a surgical decision is made. While NOM resolves ASBO and reinstates gastrointestinal physiology, it does not remove the adhesions responsible and might eventually require urgent surgical interference. Many surgeons today have been opting for non-emergent ESM in the setting of ASBO owing to its lower recurrence rates and substantial long-term health benefits.

\section{Statement of Ethics}

Written informed consent was obtained from the patient for the publication of this case report and any accompanying images.

\section{Conflict of Interest Statement}

The authors have no conflicts of interest to declare.

\section{Funding Sources}

The authors did not receive any funding.

\section{Author Contributions}

Sawsan J. Horani: writing the manuscript and data collection. Hadiel A. Kaiyasah: review and data collection. Labib S. Al-Ozaibi: review and final approval.

\section{Data Availability Statement}

All data generated or analyzed during this study are included in this article. Further enquiries can be directed to the corresponding author.

\section{References}

1 Thornblade LW, Verdial FC, Bartek MA, Flum DR, Davidson GH. The safety of expectant management for adhesive small bowel obstruction: a systematic review. J Gastrointest Surg. 2019;23(4):846-59.

2 Ten Broek R, Krielen P, Di Saverio S, Coccolini F, Biffl WL, Ansaloni L, et al. Bologna guidelines for diagnosis and management of adhesive small bowel obstruction (ASBO): 2017 update of the evidence-based guidelines from the world society of emergency surgery ASBO working group. World J Emerg Surg. 2018;13:24.
3 Khaikin M, Schneidereit N, Cera S, Sands D, Efron J, Weiss EG, et al. Laparoscopic vs. open surgery for acute adhesive small-bowel obstruction: patients' outcome and cost-effectiveness. Surg Endosc. 2007;21(5):742-6.

4 Behman R, Nathens AB, Mason S, Byrne JP, Hong NL, Pechlivanoglou P, et al. Association of surgical intervention for adhesive smallbowel obstruction with the risk of recurrence. JAMA Surg. 2019;154(5):413-20.

5 Mazzetti CH, Serinaldi F, Lebrun E, Lemaitre J. Early laparoscopic adhesiolysis for small bowel obstruction: retrospective study of main advantages. Surg Endosc. 2018;32(6): 2781-92.
6 Arung W, Meurisse M, Detry O. Pathophysiology and prevention of postoperative peritoneal adhesions. World J Gastroenterol. 2011; 17(41):4545-53.

$7 \mathrm{Mu} \mathrm{JF}$, Wang Q, Wang SD, Wang C, Song JX, Jiang J, et al. Clinical factors associated with intestinal strangulating obstruction and recurrence in adhesive small bowel obstruction: a retrospective study of 288 cases. Medicine. 2018;97(34):e12011.

8 Yang KM, Yu CS, Lee JL, Kim CW, Yoon YS, Park IJ, et al. The long-term outcomes of recurrent adhesive small bowel obstruction after colorectal cancer surgery favor surgical management. Medicine. 2017;96(43):e8316. 